\title{
analyse des vibrations engendrées par le pilonnage dynamique
}

\author{
par \\ P. Allard \\ Laboratoire Régional des Ponts et Chaussées, Aix-en-Provence
}

RESUME - Des mesures de vibrations ont été réalisées au cours d'opérations de pilonnage sur le terre-plein de Fontvieille, en Principauté de MONACO. Les principaux ouvrages ayant fait l'objet de mesure sont : un complexe sportif fondé sur semelles superficielles; une digue constituée par des caissons en béton remplis de sable et reposant sur un remblai rocheux ; des immeubles de 2 à 10 étages fondés sur le substratum.

Des mesures ont été réalisées avec : des géophones dont la fréquence propre de résonance est $4,5 \mathrm{~Hz}$; des accéléromètres à très haute sensibilité ( $1 \mathrm{~V} / \mathrm{m} / \mathrm{s} 2$ sur une gamme de fréquence de 0,1 à $1.000 \mathrm{~Hz}$ ).

Des enregistrements sur bandes magnétiques

ont permis de réaliser une analyse approfondie des vibrations : amplitude en fonction du temps et analyse spectrale sur 400 bandes de fréquences de largeur de 0,25 à $1,25 \mathrm{~Hz}$ chacune.

Ces mesures mettent en évidence que le pilonnage engendre dans le remblai des vibrations sur une plage de fréquence qui n'excède pas une quarantaine de hertz; les fréquences prédominantes se situent sur la gamme de 4 à $8 \mathrm{~Hz}$; l'atténuation des vibrations au cours de propagation dans le matériau, est à peu près uniforme en fonction de la fréquence sur la plage de 2,5 à $8 \mathrm{~Hz}$ par contre, 1 'atténuation est croissante avec la fréquence entre 8 et $40 \mathrm{~Hz}$. Une construction fondée superficiellement oscille préférentiellement suivant une direction verticale à des fréquences voisines de $1 \mathrm{~Hz}$. Des constructions fondées sur le substratum sont affectées par les vibrations de fréquences supérieures à 5 Hz, avec des caractéristiques de vibrations comparables selon les directions horizontale et verticale.

\section{INTRODUCTION}

Les remblais du terre plein de Fontvieille en principauté de Monaco sont compactés par pilonnage intensif, avec une masse de

17 tonnes lachée d'une hauteur de $25 \mathrm{~m}$.

La chute sur le remblai d'une telle masse constitue une source de vibrations qui se propagentans le remblai et viennentsolliciter des constructions et des ouvrages avoisinnants.

Nous nous intéressons en particulier à 3 types d'ouvrages de nature très différentes :

- un ensemble sportif situé sur domaine public du terre plein de Fontvieille

- la digue du bord de mer

- les immeubles du boulevard du bord de mer, les plus proches
Les mesures ont été réalisées soit pour le compte du Service des Travaux Publics de Monaco, soit pour le compte de la société "Techniques Louis MENARD" adjudicataire des travaux de compactage, dans le but de s'assurer que les vibrations engendrées par le pilonnage ne menacent pas la pérennité des ouvrages.

Notons sur ce point que toutes les opérations de compactage réalisées depuis avril 1979, tant sur le domaine public, que sur le domaine privé ont été menées en toute sécurité.

Dans l'exposé qui suit nous n'aborderons qu'un aspect particulier des phénomènes de vibrations, il s'agira de la plage de fréquence des phénomènes de vibrations en fonction des distances al point d'impact. Nous

étudierons également la fonction de transfert sur quelques dispositifs. 


\section{LES CAPTEURS}

\section{Les géophones}

Les géophones sont des capteurs qui émettent une tension électrique proportionnelle à la vitesse particulaire de vibration.

Un géophone sera utilisé sur une plage de fréquence située au-dessus de sa fréquence propre de résonance et jusqu'à 200 ou $300 \mathrm{~Hz}$.

Les géophones les plus couramment utilisés ont une fréquence de résonance de 4,5 Hz

La réponse des géophones ainsi utilisés est constante à 10 \% près sur une plage de fréquence de $5 \mathrm{~Hz}$ à environ $200 \mathrm{~Hz}$.

\section{Les accéléromètres}

Les accéléromètres sont utilisés sur une plage de fréquence située au-dessous de leur fréquence propre de résonance.

Les accéléromètres utilisés ont une sensibilité de 1 Volt $/ \mathrm{m} / \mathrm{s}^{2}$ sur une plage de fréquence de $0,1 \mathrm{~Hz}$ à $1 \mathrm{KHz}$.

\section{L'ENREGISTREMENT DES SIGNAUX}

Les signaux électrioues délivrês par les capteurs sont acheminés vers un enregistreur magnétique analogique 7 pistes modulation de fréquence. La bande passante utilisée est de 0-1250 $\mathrm{Hz}$ avec une dynamique d'enregistrement de $52 \mathrm{~dB}$.

\section{ANALYSE DES SIGNAUX DE VIBRATIONS}

L'élément principal de la chaine est constitué par un analyseur de fréquences basé sur la transformation de Fourier.

La représentation la plus courante d'un signal est de la forme $y=f(t)$.

La variable indépendante t est la durée qui s'écoule. A cette représentation, en fonction du temps, on peut faire correspondre une représentation-fréquences de la forme $\mathrm{Y}=\mathrm{F}(\mathrm{N})$ dans laquelle la variable indépendante est la fréquence dont la dimension est l'inverse d'un temps.

Ces 2 représentations d'un signal sont reliées entre elles par une relation mathématique appelée transformation de Fourier.

La fonction $Y=F(N)$ est le spectre du signal. L'analyseur utilisé (Spectral Dynamic SD 345) calcule la Transformation de Fourier sur 1.024 points, avec une fréquence d'échantillonnage du signal égale à 2,56 fois la fréquence pleine échelle. La résolution est de 400 canaux de fréquences.

Nous réaliserons dans certains cas des opérations d'intégration ou de différenciation de spectres afin de passer des accélérations aux vitesses ou inversement.
Afin d'avoir la relation de 2 spectres, nous réaliserons également quelques calculs de rapport de spectres.

Une fiche d'analyse du signal comporte en général deux diagrammes :

- le diagramme supérieur représente le signal en fonction du temps (deplacement, vitesse ou accele-ration).

- le diagramme inférieur représente le spectre de fréquence avec :

en abscisse (x), les 400 bandes de fréquence,

en ordonnée l'amplitude sur chaque bande d'analyse en échelle logarithmique.

La dernière colonne à droite du spectre représente le niveau efficace sur l'ensemble du spectre.

En bas de chaque fiche on peut lire les coordonnés $X$ et $Y$ d'un point sélectionné soit sur le signal temps, soit sur le spectre. Le point est repéré par un petit carré noir sur le diagramme concerné.

Pour caractériser un signal et son spectre de fréquence, nous proposons les paramètres suivants :

- amplitude zéro-crête maximale du signaltemps,

- fréquence d'amplitude maximale sur le spectre

- amplitude sur le canal de fréquence correspondant

- plage de fréquence de part et d'autre du canal d'amplitude maximale pour des niveaux compris entre 0 et $-6 \mathrm{db}$ sous 1 'amplitude maximale du spectre; c'est-à-dire les canaux de fréquence présentant une amplitude au moins égale à 50 \% de l'amplitude maximale du spectre.

PLAGE DE FREOUENCES DES VIBRATIONS ENREGISTREES SUR UN BATIMENT DU COMPLEXE SPORTIF

Le batiment est constitué par une ossature métallique fondée sur des semelles filantes superficielles.

Le bassin de la piscine, est mécaniquement indépendant du batiment. Il repose sur un radier superficiel en béton.

La disposition géométrique des points de mesure et des points d'impact est présentée sur le figure 1.

Les capteurs A, $K$ et $C$ sont scellés sur la semelle de fondation au pied de 2 piliers d'angle suivant la diagonale NW - SE.

Les capteurs $F$ et $J$, sont scellés au pied du bassin dans I'angle SW.

Les capteurs A, C et $\mathrm{F}$ sont des en sembles tridirectionnels de 3 géophones de fréquence de résonance 4,5 Hertz. 
Les capteurs $\mathrm{K}$ et $\mathrm{J}$ sont des éléments d'accéléromètres.

L'axe de sensibilité est vertical pour le capteur $\mathrm{K}$; et horizontal Est-Ouest pour le capteur J.

Avant le démarrage du compactage une tranchée d'environ 2 mètres de profondeur a été creusée en limite de la zone à compacter du coté du complexe sportif.

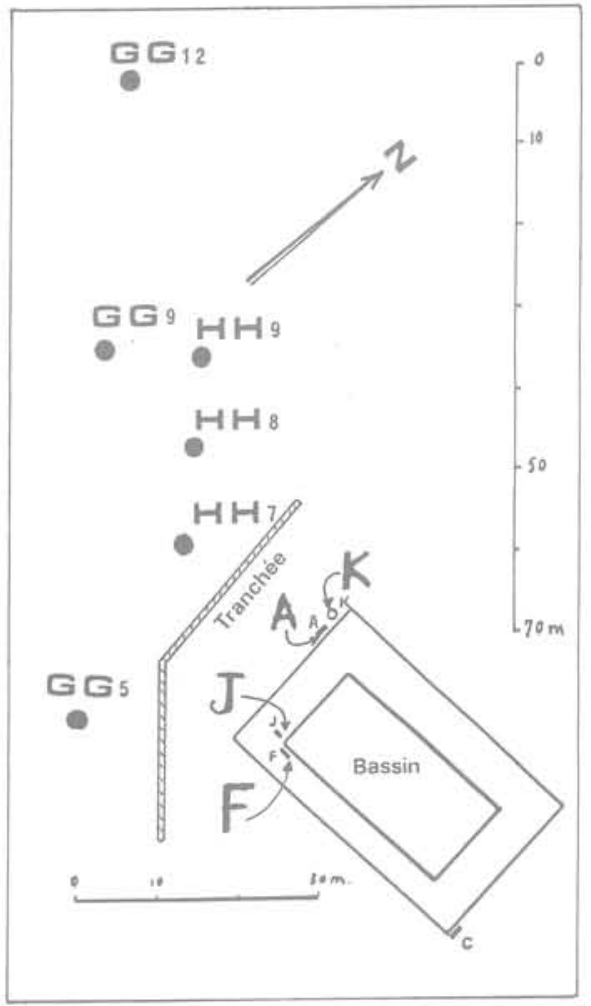

Fig. 1

Afin de sélectionner au mieux la bande totale d'analyse, nous prendrons les signaux détectés sur les accéléromètres $\mathrm{K}$ et $\mathrm{J}$, pendant le pilonnage au point GG5. Les fréquences élevées, étant généralement celles qui donnent les niveaux d'accélération les plus élevés nous aurons l'assurance de sélectionner une plage de fréquence qui renferme le maximum d'informations.

Notons que dans le dispositif de mesure, les capteurs $\mathrm{K}$ et $\mathrm{J}$ sont distants respectivement de $35 \mathrm{~m}$ et $25 \mathrm{~m}$ du point d'impact. La tranchée est d'autre part, interposée sur l'axe des trajectoires.
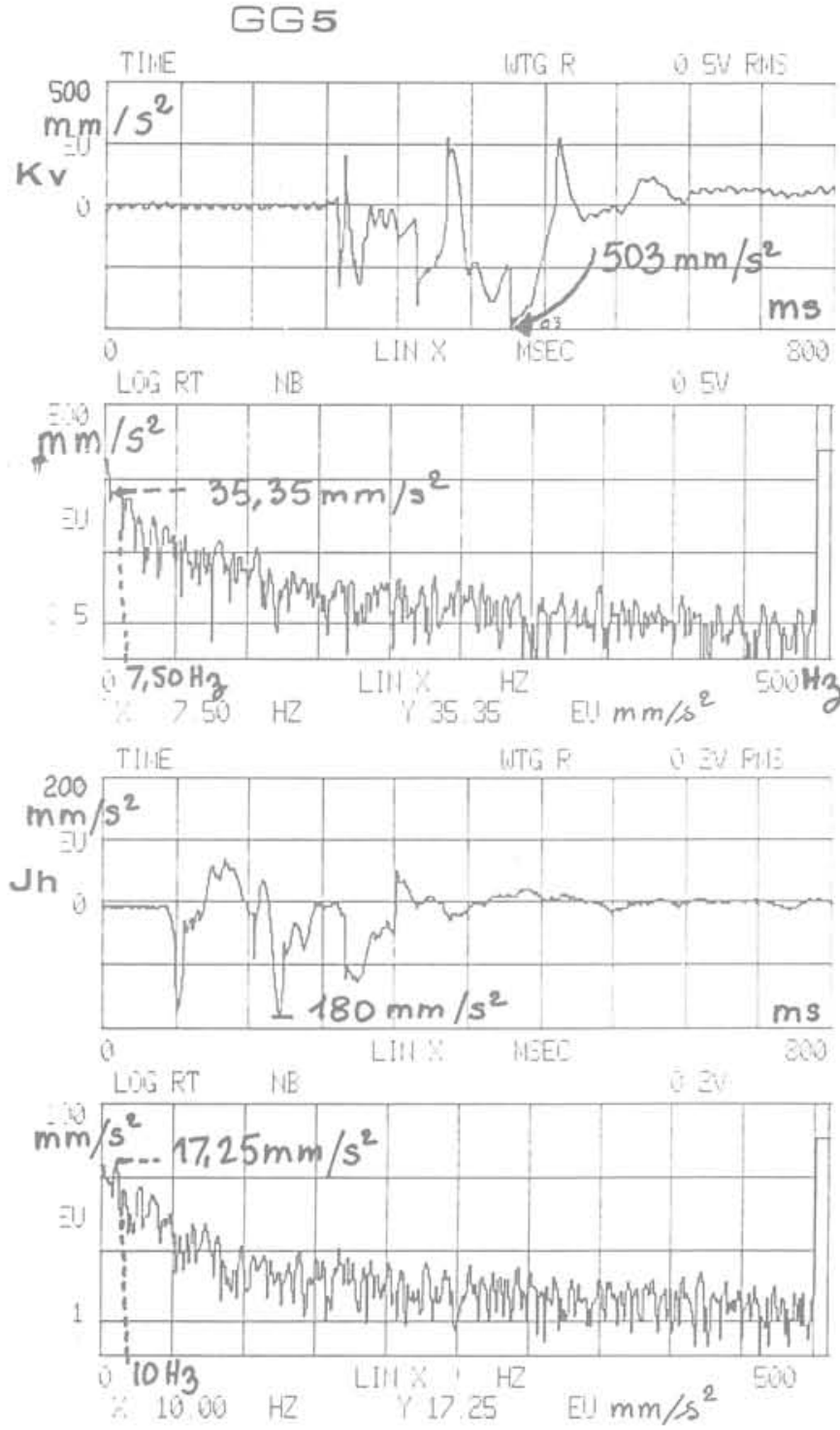

Fig. 2

Analyse sur 400 canaux de $1,25 \mathrm{~Hz}$ chacun, des signaux d'accélération sur une largeur totale de bande de 0 à $500 \mathrm{~Hz}$.

- Sur les signaux temps on note des accélérations zéro-crête maximale de $503 \mathrm{~mm} / \mathrm{s} 2$ sur $\mathrm{Kv}$ et de $180 \mathrm{~mm} / \mathrm{s} 2$ sur Jh.

- Sur les spectres on observe une décroissance des niveaux lorsque la fréquence augmente. Par rapport au canal d'amplitude maximale l'analyse sur une largeur totale de bande 0-50 Hz nous permettra d'obtenir le spectre avec une dynamique au moins égale à 20 dB en accélération sur les spectres. La dynamique sur le spectre de vitesse sera à fortiori supérieure.

Examinons par exemple le signal détecté sur le géophone vertical du capteur A (Av), ainsi que le spectre de déplacement obtenu par intégration, pour un impact sur le point GG 12 , situé à $73 \mathrm{~m}$ du capteur A, sans que la tranchée ne soit interposée sur la trajectoire (fig.3). 

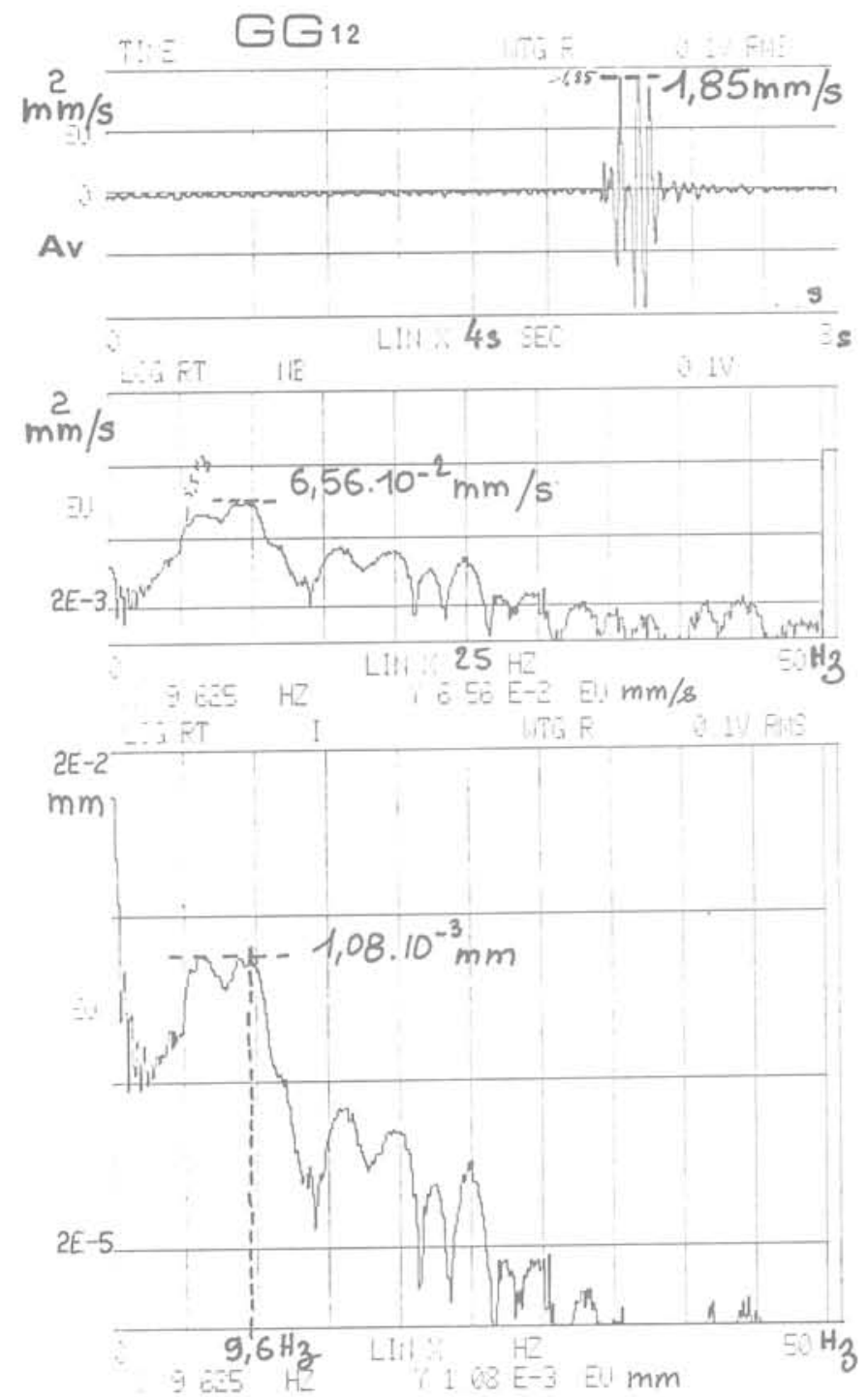

Fig. 3

Analyse sur 400 canaux de $0,125 \mathrm{~Hz}$ chacun du| siqnal vitesse sur une largeur totale de ban de $0-50 \mathrm{~Hz}$.

on observe :

- sur le signal temps une vitesse maximale zèro - crête de $1,85 \mathrm{~mm} / \mathrm{s}$,

- sur le spectre de ce signal, une raie d'amplitude maximale $y=6,5210-2 \mathrm{~mm} / \mathrm{s}$ efficace sur le canal $x=9,625 \mathrm{~Hz}$.

La valeur efficace sur la bande $0-50 \mathrm{~Hz}$ est de $0,26 \mathrm{~mm} / \mathrm{s}$.

On observe une décroissance importante des raies du spectre au-dessous de $5,5 \mathrm{~Hz}$, ce qui est en contradiction avec les spectres d'accélération observés précédemment.
Cette décroissance sur le spectre des vitesses est en fait la traduction de la courbe de réponse des géophones au-dessous de leur fréquence de résonance. Les géophones d'un modèle courant, qui ont ainsi été utilisés ne nous donnent qu'une information partielle sur le signal transmis dans le remblai lors du pilonnage dynamique. Le spectre de déplacepilonnage ànamiduent en évidence que les aéplacements sont essentiellement provoqués par les fréquences inférieures à $10 \mathrm{~Hz}$. Sur le canal $\mathrm{x}=9,625 \mathrm{~Hz}$, on note :

- une vitesse maximale sur le spectre

$y=0,065 \mathrm{~mm} / \mathrm{s}$ efficace soit près de

$0,1 \mathrm{~mm} / \mathrm{s}$ zéro-crête,

- un déplacement efficace de 1 micron.

SPECTRES OBTENUS SUR UNE MEME VIBRATION PAR DIFFERENTES TECHNIQUES

Examinons les signaux détectés simultanément par le géophone vertical AV et l'accéléromètre vertical KV, pendant le pilonnage sur le point GG5. Pour faciliter la comparaison des spectres nous prendrons le canal $7 \mathrm{~Hz}$ comme repère (fig. 4).

Spectre supérieur : spectre d'accélération obtenu par différenciation du spectre du signal vitesse délivré par un géophone dont la fréquence propre de résonance est de 4,5 Hz.

Spectre intermédiaire : spectre d'accélération sur le signal délivré par un accélérotion sur le signal délivré par un accéte la bande de fréquences.

Spectre inférieur : spectre de vitesse obtenue par intégration du spectre d'accélération KV montré au-dessus.

Observations : Au-dessous de $6 \mathrm{~Hz}$ les spectres Av et Kv ne sont pas comparables, en raison des caractéristiques même des capteurs. Le niveau d'accélération maximal est obtenu entre $0,5 \mathrm{~Hz}$ et $1 \mathrm{~Hz}$ pour $\mathrm{Kv}$.

Au-dessus de $6 \mathrm{~Hz}$ 1'allure générale des spectres est comparable. Les niveaux obtenus par différenciation sur Av sont légèrement supérieursà ceux obtenus sur l'accéléromètre $\mathrm{Kv}$ pour les canaux de fréquence compris entre 5 et $10 \mathrm{~Hz}$. Sur le canal de fréquence centrale $7 \mathrm{~Hz}$ on note par exemple :

sur le capteur Av : $\mathrm{Y}=6,21 \mathrm{~mm} / \mathrm{s}_{2}^{2}$ sur le capteur $\mathrm{KV}: \mathrm{Y}=4 \mathrm{~mm} / \mathrm{s}^{2}$

Entre 10 et $12,5 \mathrm{~Hz}$ les niveaux sont identiques.

Au-dessus de $12,5 \mathrm{~Hz}$ les 2 spectres oscillent I'un par rapport à l'autre. Le spectre de vitesse obtenu par intégration du spectre accélération Kv met en évidence que les niveaux de vitesses de vibrations vont en augmentant vers les basses fréquences. 
Sur le canal $1 \mathrm{~Hz}$ on observe une vitesse de vibration d'environ $2 \mathrm{~mm} / \mathrm{s}$ alors que sur $1 \mathrm{e}$ canal $5 \mathrm{~Hz}$, cette vitesse n'est que $0,1 \mathrm{~mm} / \mathrm{s}$.

Conclusion : Les vibrations engendrées par le pilonnage dynamique sur les semelles de fondations superficielles d'un batiment présentent un spectre de fréquences, dont les niveaux augmentent vers les basses fréquences. La plage de fréquences pour une étude complète de ces vibrations doit être d'au moins $0,5 \mathrm{~Hz}$ à une cinquantaine de Hertz.

Les accéléromètres à haute sensibilité permettent de couvrir sans problème cette plage de fréquences.

ETUDE DES FREQUENCES DE VIBRATIONS SUR LES MATERIAUX D'UN CORPS DE DIGUE

Le profil ci-dessous (fig.5) montre les positions des capteurs et du point d'impact.

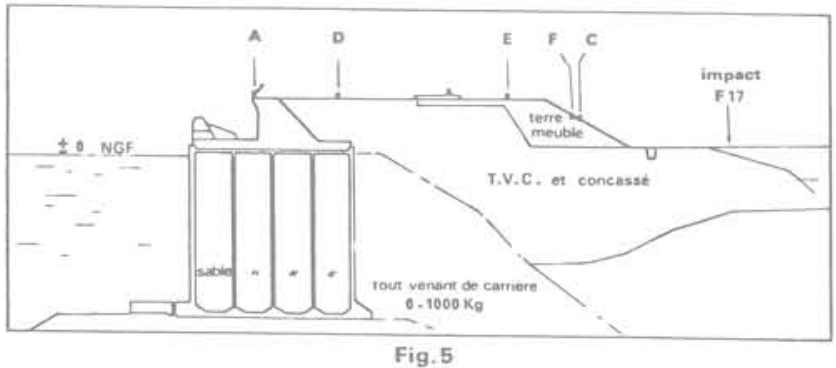

Le point d'impact F 17 est situé à environ 15 mètres des capteurs $C$ et $F$ les plus proches et à environ 45 mètres du capteur A le plus éloigné, en corniche de la digue en béton.

Le tableau qui suit donne les caractéristiques principales des signaux émis par 2 capteurs tridirectionnel à géophones $\mathrm{A}$ et $\mathrm{C}$ et 3 accéléromètres $D-E$ et $F$.

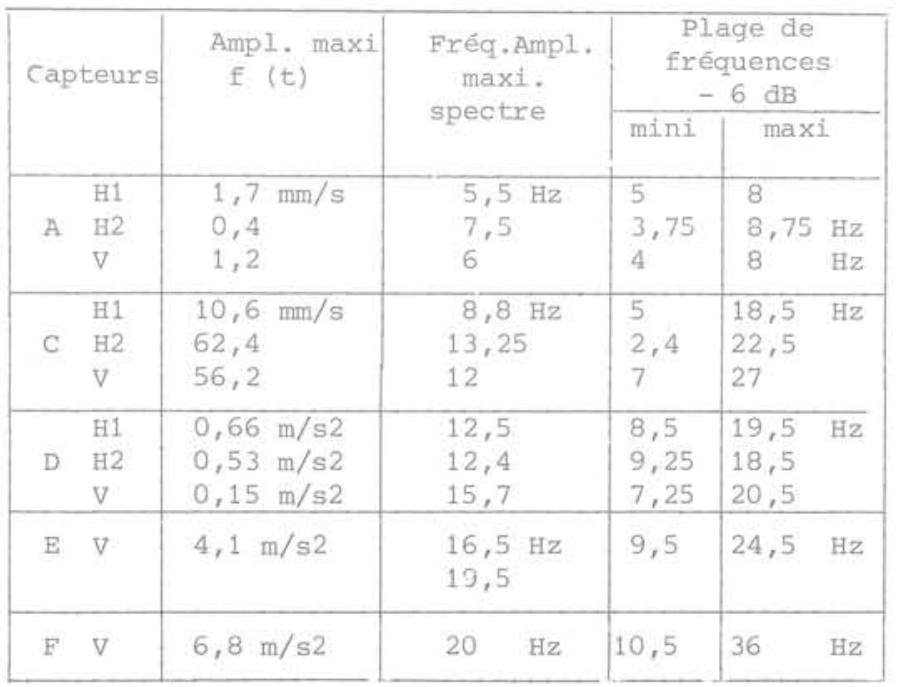
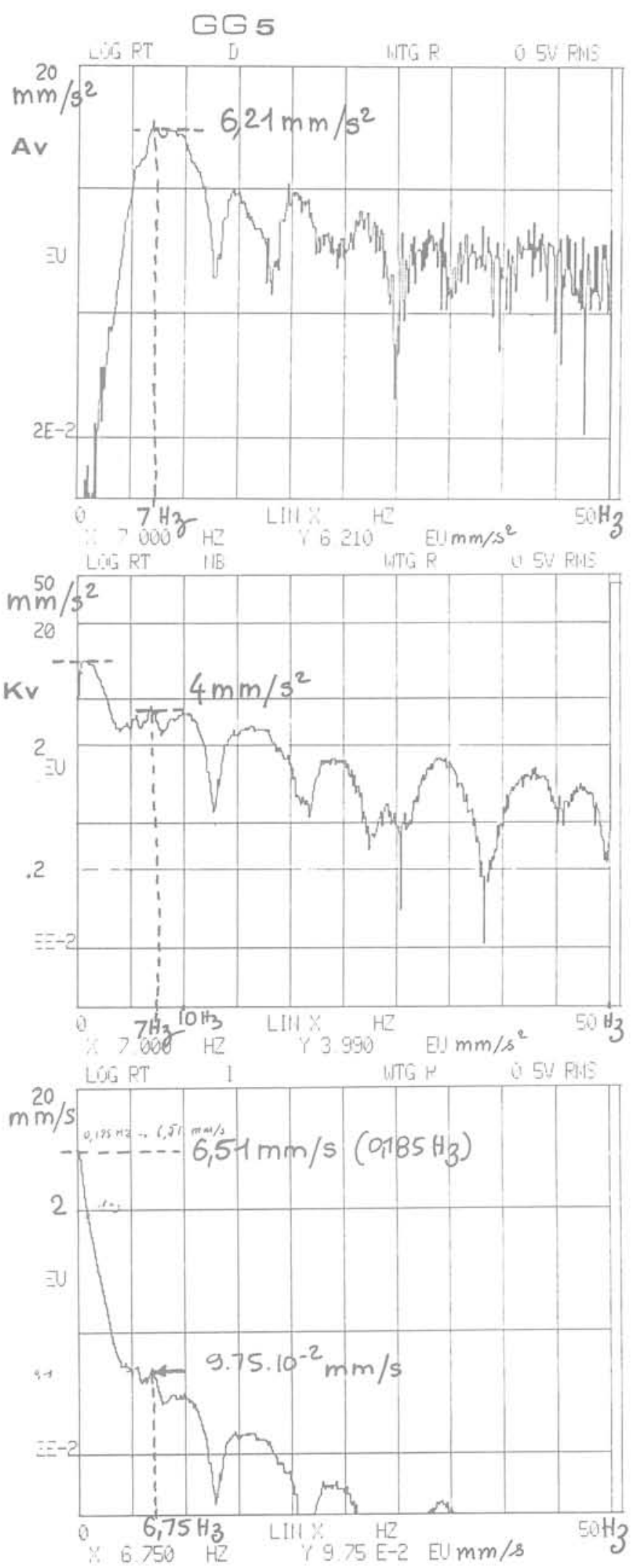

Fig. 4 
Les vibrations enregistrées sur la digue (capteur A) sont principalement contenues dans une gamme de fréquences de 4 à $8 \mathrm{~Hz}$.

Sur les capteurs les plus proches du point d'impact, nous observons une gamme de fréquences plus étalée, avec en particulier sur le géophoneCH2 une plage de fréquences de $2,4 \mathrm{~Hz}$ à $22 \mathrm{~Hz}$.

Conclusion : Les enregistrements réalisés sur les matériaux de la digue, montrent que le pilonnage engendre des vibrations sur une plage de fréquence principalement comprise entre $2,5 \mathrm{~Hz}$ et une trentaine de Hertz. Nous ne retrouvons pas les fréquences très basses de 0,5 à $1 \mathrm{~Hz}$ enregistrées sur les semelles de fondation du complexe sportif.

Des mesures analogues réalisées au pied des immeubles du boulevard du bord de mer, montre que les fréquences d'amplitude maximale vont de 6,25 à $8,75 \mathrm{~Hz}$. Les plages de fréquence à -6 dB sous les niveaux maximaux vont de 5 à $7,5 \mathrm{~Hz}$ pour les basses fréquences à 7,5 à $25 \mathrm{~Hz}$ pour les fréquences les plus élevées. Ces fréquences ont été déterminées tant avec des géophones qu'avec des accéléromètres.

ANALYSE DE LA FONCTION DE TRANSFERT SUR DIFFERENTS DISPOSITIFS DE MESURES

Pour effectuer une telle analyse nous prendrons les signaux détectés par 2 capteurs situés sensiblement dans la même direction par rapport à un point d'émission.

La fonction de transfert sera représentée par le rapport des spectres des vibrations détectées par les 2 capteurs pour un même impact.

\section{cas : Impact GG12 - Capteurs Av et CV}

Ces 2 capteurs verticuax sont scellés sur des structures analogues du batiment du complexe sportif (semelle de fondation superficielle).

La figure 8 ci-après représente :

- Les spectres des vibrations détectées par les géophones verticaux $\mathrm{A}$ et $\mathrm{C}$ distants respectivement de 72 et $115 \mathrm{~m}$ de 1 'impact GG12.

- Ia fonction de transfert obtenue par le rapport entre les 2 spectres.

Les 2 spectres présentent les niveaux les plus élevés entre 5 et $12 \mathrm{~Hz}$.

Entre 0 et $20 \mathrm{~Hz}$, on observe une atténuation croissante avec la fréquence. Les pics d'atténuation maximale se situent vers $12 \mathrm{~Hz}$ et $18 \mathrm{~Hz}$.
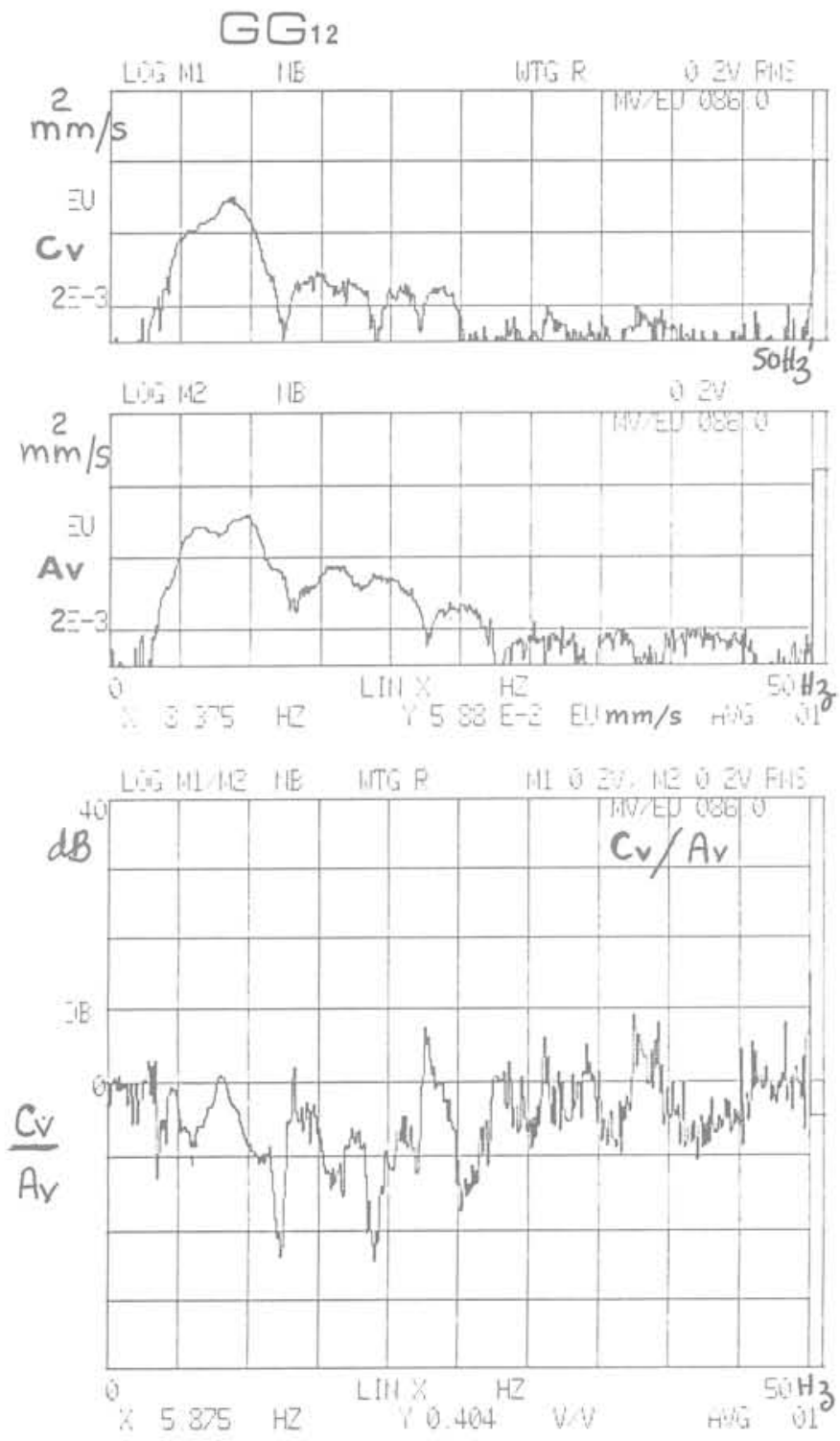

Fig. 8

On note par contre une amplification de 2 à $3 \mathrm{~dB}$ sur les fréquences de $8 \mathrm{~Hz}$ et $13 \mathrm{~Hz}$.

A $23 \mathrm{~Hz}$ l'amplification est plus forte 6 à $7 \mathrm{~dB}$, mais sur des niveaux relativement bas du spectre.

Au delà de $25 \mathrm{~Hz}$ les niveaux sont trop faibles par rapport au bruit éventuel pour donner une information fiable. (fig. 10$)$

Cas : Mesure sur le corps de digue

Accéléromètres $\mathrm{F}$ et $\mathrm{E}$ situés respectivement à $15 \mathrm{~m}$ et $22 \mathrm{~m}$ de l'impact F 17 .

Observons que, dans ce cas, où les capteurs ne sont solidaires que du terrain, à l'exclusion de toute structure, la fonction de transfert ne présente pas de pics accentués, 
comme nous l'avions observé sur le complexe sportif. Nous ne prendrons pas en considération les fréquences inférieures à $5 \mathrm{~Hz}$, dont les niveaux représentés sur les spectrés sont liés à des caractéristiques de la chaine de mesure et non au phénomène de vibrations.

Entre 5 et $8 \mathrm{~Hz}$ on note une fonction de transfert sensiblement égale à 1 .

Entre 8 et $40 \mathrm{~Hz}$, on observe globalement une atténuation de $13 \mathrm{~dB}$ soit $0,4 \mathrm{~dB} / \mathrm{Hz}$. Au dessus de $40 \mathrm{~Hz}$, les niveaux du spectre Ev sont trop faibles pour pouvoir apprécier la fonction de transfert.
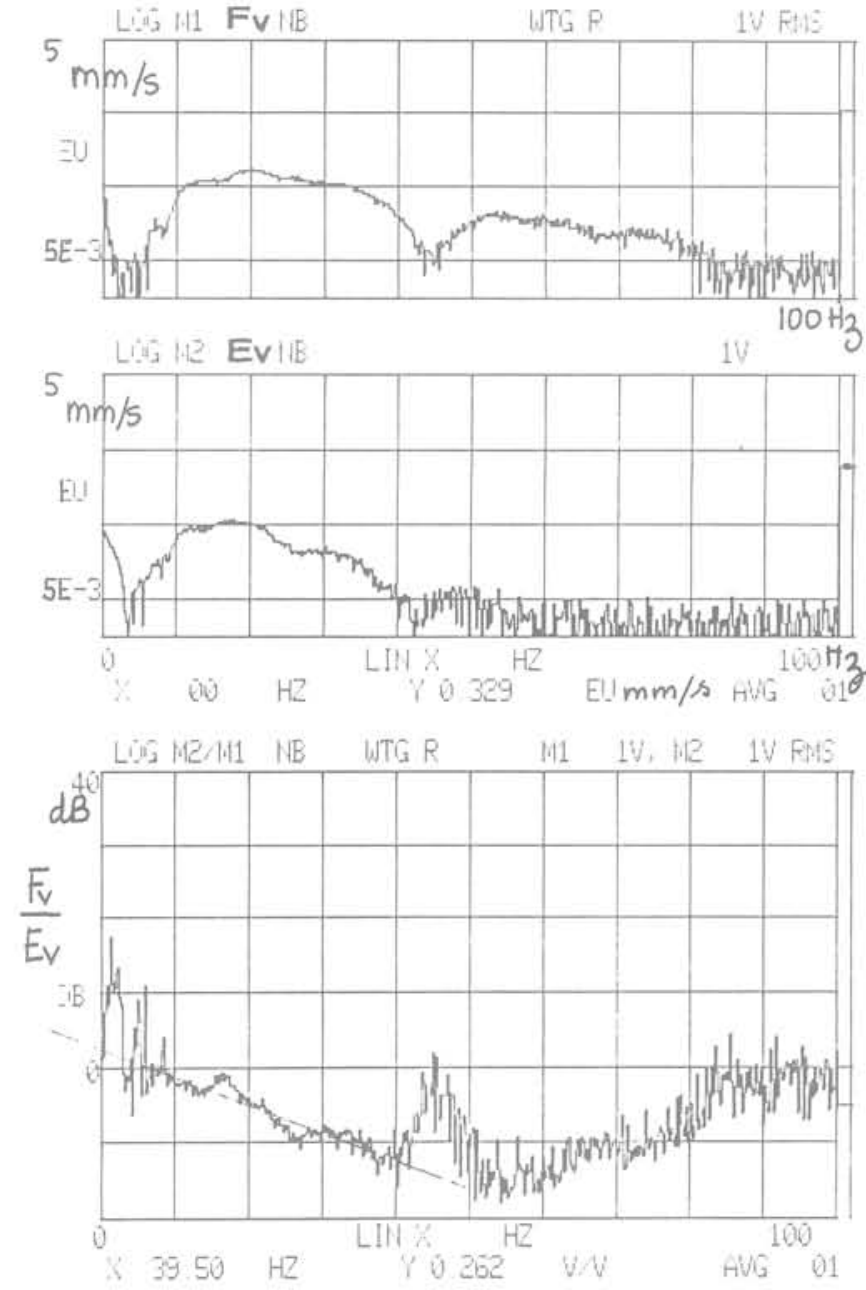

Fig. 10

Conclusion : Sur les différents dispositifs étudiés, nous retiendrons:

- une atténuation générale des niveaux de vibration lorsque les fréquences augmentent entre $8 \mathrm{~Hz}$ et $40 \mathrm{~Hz}$;

- dans le cas où le dispositif est solidaire d'une structure qui repose sur le remblai, on observe par rapport à cette pente moyenne des pics de fréquences caractéristiques.

Ainsi sur le complexe sportif on notera une transmission préférentielle sur des fréquences voisines de $15 \mathrm{~Hz}$ et un filtrage sélectif des fréquences voisines de $25 \mathrm{~Hz}$;
- dans le cas oũ le dispositif n'est solidaire que du terrain, l'atténuation est régulièrement croissante en fonction de la fréguence, elle est estimée à $0,4 \mathrm{~dB} / \mathrm{Hz}$ entre 8 et $40 \mathrm{~Hz}$.

Entre 2,5 et $8 \mathrm{~Hz}$ l'atténuation semble indépendante de la fréquence.

\section{CONCLUSIONS GENERALES}

Le compactage dynamique donne naissance dans le corps de remblai à des vibrations qui se propagent sur une gamme de fréquences de $2,5 \mathrm{~Hz}$ à $40 \mathrm{~Hz}$.

La propagation de la vibration, s'effectue avec une atténuation, indépendante de la fréquence entre 2,5 et $8 \mathrm{~Hz}$, et croissante avec la fréquence entre $8 \mathrm{~Hz}$ et $40 \mathrm{~Hz}$.

Les vibrations suivant des axes horizontaux sont souvent prédominantes, et sur une gamme et fréquences souvent très légèrement inférieure à celle des vibrations verticales.

Sous l'effet de ces vibrations une construction fondée superficiellement sur le matériau peut osciller à des fréquences voisines de $1 \mathrm{~Hz}$ préférentiellement suivant une direction verticale.

La fonction de transfert entre deux points d'une telle construction présente des fréquences singulières, qui modifient notablement la fonction de transfert dans le matériat.

Des immeubles dont les fondations sont ancrées dans le substratum, à proximité de la zone compactage, sont affectés au niveau des fondations par des vibrations sur une plage de fréquences qui ne descend pratiquement pas au dessous de $5 \mathrm{~Hz}$. Les sollicitations horizontales et verticales sont analogues.

Au cours du pilonnage en un point donné, on n'observe pas une évolution notable des caractéristiques du phénomène de vibrations dans le matériau. 